\title{
Survey on Control of Photo Sharing on Online Social Networks
}

\author{
Sahla Nazlin $\mathbf{A}^{\mathbf{1}}$, Vishnu $\mathrm{K}^{\mathbf{2}}$ \\ PG Scholar, Department of Computer Science and Engineering, CCET Engineering College, Kerala, India ${ }^{1}$ \\ Assistant Professor, Department of Computer Science and Engineering, CCET Engineering College, Kerala, India ${ }^{2}$
}

\begin{abstract}
Online Social Networks (ONS) is an important part of our everyday life...At present people are very interested to share the photo on online social networks, unluckily which is used for the purpose we not at all imagine. To check possible confidentiality outflow of a group photograph, we prepared a mechanism in which each person can take part in the decision making on the photograph posting, so everyone in the photograph must be recognized. It is important to have a professional facial recognition (FR) system that cans distinguish each person in the photograph. Haar cascade classifier and CBIR (content based image retrieval) algorithm are used in the proposed system for face detection and recognition. Once the faces are recognized from the group photograph then acceptance or reject notifications are sending automatically to each identified people within the close friend circle. The photograph will be posted if all the individuals within the friend circle are accepting the notification and it will not be posted if any of them rejects the notification. We expect that this proposed system would be very useful in protecting users' confidentiality in photo/image sharing over online social networks.
\end{abstract}

Keywords: Online Social Network, CBIR, K-Mean Clustering, Face Recognition.

\section{INTRODUCTION}

OSNS have become integral element of our everyday life and has deeply changed the way we work together with each other, data sharing, appreciation and value. photograph sharing is an beautiful feature which increases the value of Online Social Networks (OSNs).Users are allowed to post, comment, and tag a photograph without restraint, so it may disclose users' privacy . In this project, we attempt to find a solution for this issue and study the scenario when a user shares a photograph which containing persons other than himself/herself (termed cophoto for short).

At present people is very careless forgetting that once we post a photo in online, it becomes an undying record, and there are chances to use this for purposes we never expect. To prevent possible confidentiality outflow of a photograph, our designed mechanism allow each personality in a photograph be aware of the uploading photograph activity and take part in the decision making on the photograph posting. [1], [10], [11].

\section{LITERATURE REVIEW}

Photograph sharing is doubtlessly the most popular feature in online social networks. Unfortunately, casual photograph posting may reveal privacy of persons in a posted photograph. In this section we discuss about the security issues and the available prevention methods related to group photo uploading, about the Haar cascade classifier, which is used for face detection and explain CBIR algorithms used for the face recognition system.
Tagging photograph is one of the popular feature of many social network sites that allows people to annotate uploaded photograph with those who are in them, and linking the photograph to each person's profile. Actually this is a serious privacy leakage issue. To solve this now Facebook added the new security option to avoid this confidentiality leakage issue related Tagging photograph, people can set "review the tag before appear the time line option this a good method to prevent this privacy leakage issue, still this security option can prevent the co- photo owner's privacy in a very limited level [2].

A face recognition algorithm can be divided into the following functional modules: a face image detector finds the locations of human faces from a normal picture against simple or complex background, and a face recognizer determines who this person is.

TABLE 1. Various face detection methods

\begin{tabular}{|l|l|l|}
\hline YEAR & AUTHOR & METHODS \\
\hline 1973 & Kande & First automated system \\
\hline 1987 & $\begin{array}{l}\text { Sirovich \& } \\
\text { Kirby }\end{array}$ & $\begin{array}{l}\text { Principal Component } \\
\text { Analysis }\end{array}$ \\
\hline 1991 & Turk\& Pentland & Eigenface \\
\hline 1996 & $\begin{array}{l}\text { Etemad \& } \\
\text { Chellapa }\end{array}$ & Fisherface \\
\hline 2001 & Viola\& Jones & AdaBoost+HaarCascade \\
\hline
\end{tabular}

Both the face detector and the face recognizer follow the same framework; they both have a feature extractor that 
transforms the pixels of the facial image into a useful vector representation, and a pattern recognizer that searches the database to find the best match to the incoming face image.

There is various face detection methods available now, a few is shown in the table 1. Face detection algorithms detects faces and determines the precise face area and exposure conditions. Automatically crops images or sets the proper exposure conditions for optimum results. These are First automated system,PCA,Eigenface, Fisherface and AdaBoost+HaarCascade.Outof this Haar cascade classifier is a well used classifier for face detection[7],[11].

Haar cascade classifier is a well used classifier for face detection. It is trained with a lot of face images and non face images.Initillay more than $160000+$ features values are used within a detector at $24 \times 24$ base resolutions which need to be calculated. For this Haar features shown in the Fig. 1 is used. Each feature is a single value which is calculated by subtracting the sum of pixels in white rectangle from the sum of pixels in black rectangle. To reduce the computation cost used the concept integral image. Most of the features among 160000+ features are not relevant so used Adaboost algorithm to find out the relevant features. To do this every features apply to training image and elected the features with minimum error rate .After these are found a weighted combination of all features are used $n$ evaluating and deciding the given window has a face or not Each feature is called a week classifying Adaboost construct a strong classifier as a linear combination of these week classifier

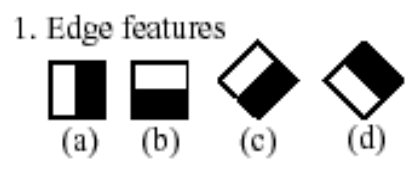

2. Line features
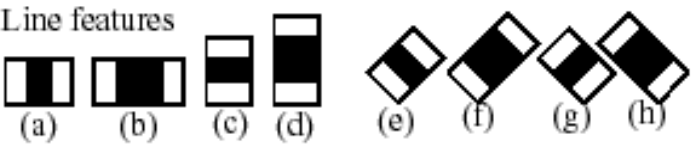

3. Center-surround features

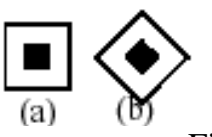

Fig.1 Haar features

Cascade classifier composed of different stages and each stage has certain no of features.Eachstage is used to determine a given sub windows face or not. The sub window is immediately discarded if it is not a face. Face detection using Haar cascade classifier is very fast, it has an efficient feature selection still there will be error or miss classification. $100 \%$ face detection cannot ensure using cascade classifier [7].

Using face annotation for effective management of personal photos in online social networks (OSNs) is currently of considerable practical interest. In this paper, it is proposed a novel collaborative face recognition (FR) framework, improving the accuracy of face annotation by effectively making use of multiple FR engines available in an OSN. In particular, our collaborative FR framework consists of two major parts: selection of FR engines and merging (or fusion) of multiple FR results. The selection of FR engines aims at determining a set of personalized FR engines that are suitable for recognizing query face images belonging to a particular member of the OSN. For this purpose, we exploit both social network context in an OSN and social context in personal photo collections. In addition, to take advantage of the availability of multiple FR results retrieved from the selected FR engines, we devise two effective solutions for merging FR results, adopting traditional techniques for combining multiple classifier results. Experiments were conducted using 547,991 personal photos collected from an existing OSN. Our results demonstrate that the proposed collaborative FR method is able to significantly improve the accuracy of face annotation, compared to conventional FR approaches that only make use of a single FR engine. Further, we demonstrate that our collaborative FR framework has a low computational cost and comes with a design that is suited for deployment in a decentralized OSN. 100\% accuracy cannot be ensured [11].

Facial recognition technology once seemed like something out of the movies, but it is increasingly being incorporated into our everyday lives. Both public and private sector organizations are incorporating facial recognition into products and services to create substantial benefits for consumers. As with any new technology, innovative capabilities can present new or expanded privacy risks. Facial recognition data is personal and sensitive, making privacy an important challenge for companies. Commercial use of facial recognition technology raises many of the same security concerns applicable to sensitive personal information generally. social networks and other large databases of identified individual images could increasingly become the targets of access by unauthorized individuals, leading to consumers' facial recognition data being used in ways that consumers cannot anticipate or control, and without their knowledge.

CBIR, Content Based Image Retrieval has been a significant area of research in the last few decades. A retrieval mechanism using color and texture is being proposed here. Depending on the characteristic of the image texture, it can be represented by multi wavelet transform. Through the combination of Multi wavelet decomposition and color correlogram it is possible to increase the number of features and can improves the retrieval accuracy. To support the efficient and fast retrieval of similar images from image databases, feature extraction plays an important role. The technique used for comparing images plays the fundamental ingredient of content based image retrieval. The main advantage of wavelet decomposition is that it yields a large number of 
sub bands. A major disadvantage of this technique is its limitation in feature set. The problem of retrieving desired image from huge database is a major problem. [3], [5].

Existing system explain the image retrieval using CBIR with Haar wavelength transformation. System Architecture of CBIR system is shown in Fig.2.It has mainly two phases, indexing and searching. Indexing includes decomposition, feature extraction and clustering and another one is searching for a query image. It is an image retrieval technique using Modified Haar Wavelet Transform and K Means Clustering First process is image training including decomposition, feature extraction and clustering. In the searching phase the most similar images to the query image [12], from the cluster files can be retrieved using CBIR image retrieval algorithm [8].

\section{A. CBIR Algorithm}

Purpose: Retrieving most similar image to input image. Input: Query image.

Output: Most similar image to the input image.

Procedure:

\{

Step 1: The input image.

Step 2: Extract the feature vector for the input image

Step 3: Calculate the weighted features vectors for the input image.

Step 4: Calculate the distance between the input image and the centroid of each K-mean cluster and find the smallest distance.

Step 5: Calculate the distance between the input image and the image in the cluster that has the smallest distaance with the input image.

Step 6: Retrieve the image that is more similar to the input image.

A Haar Wavelet transform decomposes an image into two components: average and difference. Each colour in the image can be represented by considering the pixels as a point in space and from these matrices for each Red; Green and Blue components of RGB are constructed. This is then decomposed into four sub-matrices through row and column transformations. Fill the first half of the array with averages and second half of the array with differences, this steps are repeated for both row and column. Once the process is completed, the matrix gets decomposed into four sub-matrices each of dimension (number of rows/2) x (number of columns/2).

Feature vectors can be constructed using mean and variance... If we got square matrix $A$ and sub matrix $A_{i}$ from it, then calculate F-norm based on F-norm theory. A typical content-based retrieval the system automatically extracts visual attributes and saves to the cluster file. Image retrieval the system returns all relevant images to query image based on minimum "Euclidean distance [6]. CBIR algorithm is best for face recognition, it is simple and fast. $100 \%$ face recognition cannot ensure using CBIR algorithm.

\section{PROBLEM DEFINITION}

In a typical CBIR system, low- level visual image features that is colour, texture, and shape are automatically extracted for picture descriptions and indexing purpose. To search for desirable images, a user need to presents an image as an example of similarity, and the system returns a set of similar images based on the extracted features the main issue with CBIR system is the time of image retrieval in almost all CBIR systems depends in a large degree on the number of images in the cluster file. Two main issues with CBIR systems are efficiency and accuracy. Hence, an effective CBIR system needs to have an efficient search mechanism and also accurate set of features. Out of all the mechanisms used in CBIR, kmeans clustering is found to have better efficiency and accuracy.

\section{PROPOSED SYSTEM}

The proposed system uses Haar cascade classifier for face detection and CBIR algorithm for image training and face recognition. Here the advantages of Haar cascade classifier and CBIR algorithm are applied in the social networking sites for better performance. To curb the privacy leakage, we proposed to enable individuals potentially in a photo to give the permissions before posting a co-photo. We designed a privacy-preserving Content based image retrieval FR system to identify individuals in a co-photo. The proposed system is featured with low computation cost and confidentiality of the training set.The proposed system added time limit for notification acceptance, and SMS notification to inform the co-photo owner about the time limit for notification. We expect that our proposed scheme be very useful in protecting users' privacy in photo/image sharing over online social networks.

\section{CONCLUSION}

Photograph sharing is one of the most popular features in online social networks such as Facebook. Lamentably, careless photograph posting may expose security of humans in a posted photograph. To control the security spillage, we proposed to empower people possibly in a photograph to give the consents before posting a cophotograph our future work could be the way to move the proposed plans to individual mists like Dropbox and/or icloud.and the same face recognition concept can be used to prevent privacy leakage while uploading videos.

\section{ACKNOWLEDGMENT}

I express my sincere gratitude to my guide Mr. Vishnu k, Assistant professor of Computer Science and Engineering, for his valuable guidance and encouragement for the paper work. I acknowledge with grateful thanks the authors of the references and other literatures in this survey. 


\section{REFERENCES}

[1] KAIHE XU, YUANXIONG GUO, LINKE GUO, YUGUANG FANG AND XIAOLIN LI," MY PRIVACY MY DECISION: CONTROL OF PHOTO SHARING ON ONLINE SOCIAL NETWORKS", IEEE TRANSACTIONS ON DEPENDABLE AND SECURE COMPUTING, VOL.14, PP.199-210, 2015.

[2] A.Besmer, H. Richter Lipford "Moving beyond UN tagging: photo privacy in a tagged world. In proceedings of the SIGCHI" Conf e fence on Human Factors in computing Systems, Vol.10, pp.1563-1572, 2015

[3] Y. C. C.H. Lin, R.T. Chen "A smart content-based image retrieval system based on color and texture feature," Sciencedirect journal on Image and Vision Computing, Vol.27, pp. 658-665, 2009.

[4] P.V.N Reddy, K.Satya Prasad," Colour and Texture Features for Content BasedImage Retrieval", International Journal on Computer Application and Technology, Vol.4, pp.1016-1020, 2011.

[5] Besmer, Andrew and Heather Richter Lipford, "Moving Beyond Untagging, "Photo Privacy in a Tagged World." CHI '10: Proceedings of theSIGCHI Conference on Human Factors in Computing Systems", Vol.10, pp.1563-1578, 2010.

[6] Pansur M.A and P.s Malge , “Image Retrieval Using Modified Haar Wavelet Transform and K Means Clustering”, International Journal of Emerging Technology and Advanced Engineering, Vol.3, pp. 89-93, 2014.

[7] P.Viola and M.Jones, "Rapid object detection using a boosted cascade of simple features, "IEEE Computer society Conference on Computer Vision and Pattern Recognition”, Vol .2 , pp.8-14, 2001.

[8] Divyalaxmi , R. Nampalli and Trupti Dange," A Survey Paper on Photo Sharing and Privacy Control Decisions , "International Journal on Recent and Innovation Trends in Computing and Communication ,Vol.3, pp.6327-6331, 2015.

[9] Nishant Singh, Shiv Ram Dubey, Pushkar Dixit, Jay Prakash Gupta,"Semantic Image Retrieval by Combining Color, Texture and Shape Feat ures," International Conference on Computing Sciences, Vol.3, pp.116-120, 2012.

[10] K. Thomas, C. Grier and D. M. Nicol, "Unfriendly: Multi-party privacy risks in social networks", Proc. 10th Int. Symp. Privacy Enhancing Technoogies1, Vol.4, pp.236-252, 2010.

[11] J. Y. Choi, W. De Neve, K. Plataniotis, and Y.-M. Ro., "Collaborative face recognition for improved face annotation in personal photo colle ctions shared on online social networks, " IEEE Transactions on Multimedia, Vol.13, pp.14-28, 2011.

[12] K. Choi, H. Byun, and K.-A. Toh.” A collaborative face recognition framework on a social network platform",. 8th IEEE International Conf erence on Automatic Face and Gesture Recognition, Vol.8, pp. 1-6, 2008.

\section{BIOGRAPHIES}

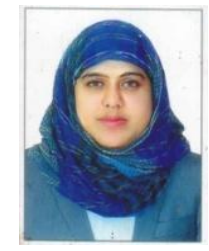

Kerala, India.

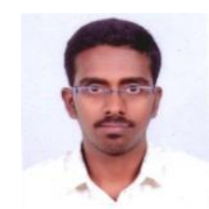

Coimbatore.

Sahla Nazlin A received B-Tech degree in Computer Science Engineering from Government Engineering College Palakkad, Kerala, in 2008. Now doing MTech in Computer Science \& Engineering from Cochin College of Engineering andTechnology Valanchery, Malappuram,

Vishnu $\mathbf{K}$ received $B-T e c h$ degree in Information Technology from College of Engineering and Management, Punnapra, Alappuzha, Kerala in 2012 and received $\mathrm{ME}$ in Computer Science Engineering from Anna University Regional Campus, 